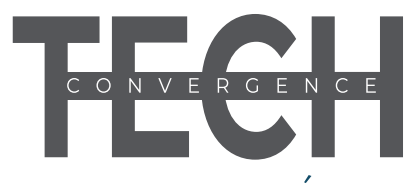

\title{
SOLUCIÓN DE SOBREVIVENCIA Y CRECIMIENTO PRODUCTIVO Y ECONÓMICO EN ÉPOCAS DE LOS MICROORGANISMOS PATÓGENOS, INICIADA POR EL COVID 19 Y CUARTA REVOLUCIÓN INDUSTRIAL
}

\author{
Hugo Fernando Segovia Albarracín \\ GRUSAM: Centro de Investigación, Tecnologías y Servicios \\ fernando.segovia@grusamse.com \\ (D) https://orcid.org/0000-0002-3149-925X
}

Fecha de recepción:

Fecha de aceptación:

\section{RESUMEN}

Del 82,5 al $93 \%$ de las organizaciones se mantienen en una crisis de rentabilidad social y económica, causada por cierres de los sistemas laborales y productivos, caídas de la demanda y del PIB (Producto Interno Bruto) en Latinoamérica (promedio 6,3\%-10\%). Estas se ven transparentadas en la emergencia ambiental, humana, y económica, iniciadas con el COVID 19, que ha sorprendido a las organizaciones con estructuras débiles y sistemas funcionales de control de la variabilidad de riesgos, improvisación, y bajo rendimiento de recursos existentes en sus procesos.

Bajo esta difícil realidad, en este artículo se propone una solución científica: Sacrificar financieramente la grasa o recursos innecesarios; mientras que el capital humano de las partes interesadas en superar la crisis da valor al diseño, ejecución y monitoreo de estrategias, y

\section{ABSTRACT}

From 82.5 to $93 \%$ of the organizations remain in a crisis of social and economic profitability, caused by closings of the labor and production systems, drops in demand and GDP (Gross Domestic Product) in Latin America (average 6.3 $\%-10 \%)$. These are revealed in the environmen- potencializan en el tiempo el recurso existente. Un equipo de expertos con aptitudes académicas, emocionales, mentales y sociales, estructura y fortalecen sistemas de alto rendimiento de recursos e inversiones con herramientas estadísticas y de análisis, trabajo colaborativo, transferencia de conocimiento y tecnologías desconcentradas de internet. Estas herramientas permitirán hacer frente, con alineamiento de objetivos, al control integral de riesgos y su variabilidad en el tiempo, mientras se fortalece con salud, seguridad ocupacional, control riesgos y cultura organizacional la calidad lean six-sigma de la investigación, planificación, desarrollo e innovación. Estas elevan el rendimiento de la rentabilidad social y económica y, por consiguiente, de la productividad y competitividad que fortalecen la micro y macroeconomía de sus inversionistas y equipos de trabajo.

Palabas clave: alto rendimiento, rentabilidad, desarrollando, variabilidad del riesgo

tal, human, and economic emergency initiated with COVID 19, which has surprised organizations with weak structures and functional systems of risks variability control, improvisation, and low performance of existing resources in their processes.

Under this difficult reality, this article proposes a scientific solution: Financially sacrifice fat 
or unnecessary resources; while the human capital of the parties interested in overcoming the crisis gives value to the design, execution and monitoring of strategies and potentiates the existing resource over time. A team of experts with academic, emotional, mental, and social skills structure and strengthen high-performance systems of resources and investments with statistical and analytical tools, collaborative work, knowledge transfer, and decentralized Internet technologies. These tools will make it possible to face, with objective alignment, com- prehensive risk control and its variability over time, while strengthening the six-sigma quality of research, planning, development with occupational safety, risk control and organizational culture and innovation. These increase the performance of social and economic profitability and, consequently, of productivity and competitiveness that strengthen the micro and macroeconomics of its investors and work teams.

Keywords: high performance, cost effectiveness, developing, risk variability

Forma sugerida de citar: Segovia Albarracín, H. F. (2020). Solución de sobrevivencia y crecimiento productivo y económico en épocas de los microorganismos patógenos, iniciada por el COVID 19 y cuarta revolución industrial. Convergence Tech Revista Científica. 4(1), 49-58.

\section{INTRODUCCIÓN}

La problemática ambiental, humana, productiva y económica que se identifica con mayor gravedad y frecuencia a partir de la crisis ambiental, humana y económica en épocas de los microorganismos patógenos iniciada por el COVID 19 y la cuarta revolución industrial. Es claro; a nivel global del 82,5 al 93\% de organizaciones privadas y públicas, se mantienen en crisis de su rentabilidad social y económica; así como ha caído de 6,3 al $10 \%$ en promedio el PIB Producto Interno Bruto de los países latinoamericanos; y en un alto porcentaje la tasa de empleo, según reportes de organismos competentes internacionales. Transparentadas en la emergencia ambiental, humana y económica que vive la sociedad tras el aparecimiento del COVID 19. Que ha sorprendido repentinamente a la sociedad y a todas las organizaciones del mundo con débiles estructuras funcionales y sistemas de control de la variabilidad de riesgos, improvisación, mantenimiento de bajo rendimiento o sub-utilización de los existentes recursos económicos, materiales, herramientas, insumos, infraestructura de sus procesos.

Así como por desperdiciar, mal utilizar y/o poner en riesgo las aptitudes, intelectuales, físicas, sociales, emocionales de su capital humano; en especial del sistema de seguridad y salud ocupacional y organismos paritarios de los entornos laborales, que son obligatorios por mandatos de ley y normas internacionales de la OIT Organización Internacional del Trabajo sin excepción; que desencadenan olas de corrupción y desperdicios eco- nómicos sin costo beneficio real para los inversionistas y partes interesadas, por falta de conocimiento, aptitudes y los débiles sistemas estratégicos integrales de gestión adoptados; que son incapaces de medir y reportar estadísticamente en tiempo real el desempeño de cada proceso, para la toma de acciones correctivas y de mejora de su capital humano.

La vulneración de toda buena intención; conllevan además a la acumulación variable de problemas y riesgos, que atentan con la integridad a la vida, salud y patrimonio del ser humano; incluso con indicios de presunciones de responsabilidad administrativa, patronal, civil, penal. Por principios constitucionales y legales de desconocimiento, omisión, acción, responsabilidad, corresponsabilidad. Materializadas en la fragilidad del representante legal, inversionistas, equipos de trabajo y trabajadores en general a sufrir acciones de insolvencia, quiebra, delitos, corrupción, accidentes, muertes, enfermedades ocupacionales.

\section{METODOLOGÍA}

El presente ensayo se sustenta en una investigación exhaustiva, con intervención analítica del investigador; por lo que se consagra en experimental de tipo prospectivo, con actividades, tareas y datos primarios planificados, provenientes de varias mediciones como estudio longitudinal de variables realizadas; con sesgos controlados por el propio investigador, que al momento de ejecutarse debe cumplir con requisitos, protocolos, manuales o pro- 
cedimientos correspondientes a cada especialidad como variable de estudio, que persiguen un escenario apuesta o ideal con estudios aplicativos; cuya intervención es para mejorar las condiciones de la población objetivo.

Los estudios relacionales que plantean descubrir los factores de riesgo, son observacionales; porque en ningún caso se expondría intencionalmente a las personas, organizaciones o procesos involucrados; a potenciales y agresivos factores cambiantes de riesgo, que pueden o no transformarse en el tiempo, en generadores de pérdidas humanas, materiales y/o económicas.

Técnicas que soportan la metodología:

- Técnicas de selección de talentos por aptitudes laborales y tareas (Aptitudes: Académica, mental, emocional, física, social)

- Técnicas metrológicas con control estadístico de procesos de los sistemas integrados de gestión, basadas en estándares internacionales ISO - Lean six sigma

- Técnica del KHMCR Know How Mejoramiento Continuo Radical

\section{RESULTADOS}

Mientras el capital humano de las partes interesadas en superar la crisis, dan valor al diseño, ejecución y monitoreo de estrategias y potencializan en el tiempo el recurso existente; se suman al equipo, profesionales con aptitudes académicas, mentales, emocionales, físicas y sociales en el control integral de la variabilidad de riesgos, con herramientas estadísticas y de análisis; para estructurar y fortalecer sistemas de alto rendimiento de recursos e inversiones, con trabajo colaborativo, transferencia de conocimiento y tecnologías desconcentradas de internet. Que hagan frente con alineamiento de objetivos, al control integral de riesgos y su variabilidad en el tiempo. Mientras se fortalece con salud, seguridad ocupacional, control riesgos y cultura organizacional, la calidad lean six sigma de la investigación, planificación, desarrollo, innovación. Que elevan el rendimiento de la rentabilidad social y económica, por consiguiente, de la productividad, competitividad que fortalecen la micro y macro economía de sus inversionistas y equipos de trabajo.
Considerando que:

ALTO RENDIMIENTO = Pensamiento y Acción Estratégica + Requisitos de Clientes y Partes Interesadas + Requisitos Legales Aplicables + Normas Internacionales OIT, OMS, OMC, FAO, CAN, ISO + Capital Humano + Estrategias de Desarrollo Organizacional + Métodos y Técnicas Científicas y Empíricas con resultados + KHMCR BI GRUSAM, Sistema Operativo de Organizaciones y Procesos de Alto Rendimiento, Software Libre Desconcentrado.

Es necesario RECONOCER ésta propuesta de acción estratégica de desarrollo de organizaciones y procesos, partiendo de una interrogante

¿Qué se necesita para salir de ésta crisis ambiental, humanitaria y económica, agravada por factores biológicos como el caso del COVID 19?

Es inicialmente necesario reconocer, que la actual crisis que atraviesa el mundo, es de tipo ambiental, cultural y humano que acarrean problemas de rentabilidad social y económicos; y por consecuencia generan efectos en la productividad, competitividad y sistema económico. Por ésta razón, es necesario que la información y el conocimiento disponible en toda la sociedad se lleve a la acción y generen resultados de alto rendimiento en beneficio de las partes interesadas; sin discriminación, riesgos, sobornos, ni corrupción; luego de solucionar secuencialmente los problemas ambientales, humanitarios, productivos y de rentabilidad social y económica de la sociedad de interés.

En éste sentido, basados en procedimientos matemáticos y estadísticos se propone para evitar divergencias de opiniones o criterios: implementar en todo sistema laboral con capital humano en plenas aptitudes, el control integral de riesgos y su variabilidad, con herramientas estadísticas y de análisis. Lo cual, garantiza la calidad de vida de procesos, cadenas de valor, organizaciones, stakeholders.

Es así como la salud y seguridad del capital humano y del trabajo. Porque mientras la salud y el trabajo son asegurados, se definen el nivel de productividad. Todo este juego de variables en secuencia, determinan la condición del bienestar y economía. En éste sentido; al ser 
el bienestar y la economía, el resultado de la calidad de la salud y seguridad de: inversionistas, capital humano y del trabajo. Es fácil comprender que a menor riesgo en cualquier actividad; en especial de inversionistas y del talento humano de los sistemas laborales, mayor es la calidad de vida y competitividad; que aseguran en el tiempo mejora de la rentabilidad social y económica de forma sustentable y sostenible. Por consecuencia hay crecimiento económico.

La solución, en síntesis. - Mientras el capital humano de las partes interesadas en superar la crisis ambiental, humanitaria y económica; que actualmente asechan a los ecosistemas dan valor al diseño, ejecución y monitoreo de estrategias, desde el cuadro de mando integral o sus componentes; profesionales selectos con aptitudes a cada proceso, hacen frente al control integral de la variabilidad de riesgos, seguridad y salud ocupacional. Desde la planificación, acción, verificación, actuación y mejora. Estructuran y fortalecen sistemas de procesos y organizaciones de alto rendimiento de recursos e inversiones; bajo la dirección estratégica de un experto en el área y un equipo humano auxiliar multidisciplinario con plenas aptitudes en actividades y tareas complementarias.

Esta propuesta es generadora del aparecimiento de un nuevo sistema de trabajo desconcentrado y desarrollo de la productividad, con riesgos controlados en toda su estructura y acorde al contexto ambiental, humano y económico actual. Como el caso de la industria de la mentefactura combinada con la manufactura en óptimas condiciones de seguridad y salud ocupacional; aprovechando todos los recursos existentes en su entorno, sin caotizar sistemas. En donde por efecto surgirán con alta calidad de vida y desempeño. Sistemas estructurados como: el educativos, laboral, emprendimientos, humanitarios, sociales, políticos de calidad, tendientes a cometer 3,4 errores por cada millón de procedimientos en sus tareas que equivale al $100 \%$ de rendimiento y $0 \%$ de riesgos y corrupción. Este gran efecto generará el aparecimiento en todos los sectores, de muchos productos de demanda interna o global con alto nivel de competitividad. Que será las causas para que se incrementen la rentabilidad social y económica a todos sus inversionistas y trabajadores que fortalecerán la micro y macro economía.
En palabras sencillas, cuando hay bienestar, seguridad y salud ocupacional, existe con trabajo colaborativo alta calidad en productos y servicios competitivos; así como bajo nivel de riesgos; en consecuencia, alta rentabilidad social y económica. Por el contrario, cuando existe un mediocre o despreocupado sistema de gestión de seguridad y salud ocupacional, existe alto nivel de riesgos, incluso para sus inversionistas, asesores y equipos de trabajo; así como baja calidad en los productos y servicios que se ofrecen a las partes interesadas. El resultado final es una profunda crisis social y económica.

En éste sentido, como es de conocimiento general que:

El único camino recto y sin pérdidas de tiempo ni recursos para superar etapas de crisis, problemas y riesgos; es elevar al máximo rendimiento los recursos existentes. Así como controlar secuencialmente en orígenes los riesgos, problemas y desperdicios de mayor índice de gravedad, de manera sostenible en el tiempo. Con un sistema integrado de gestión de la calidad y anti soborno generado por el trabajo colaborativo, que persigan bajo requisitos de las partes interesadas, estamentos legales y estándares internacionales; ser altamente rentables social y económicamente para inversionistas y partes interesadas. Aclaro, esta obligación es única y exclusiva para procesos y organizaciones públicas y privadas; así como para profesionales y/o personas naturales o jurídicas de alto rendimiento. En tal razón, se utilizan herramientas estadísticas y de análisis, Los demás toman sus propias decisiones de sobrevivencia.

\section{DISCUSIÓN}

\section{KNOW HOW - SABER HACER}

¿CÓMO LLEVAR A LA PRÁCTICA LA IMPLEMENTACIÓN DEL CONTROL INTEGRAL DE RIESGOS Y SU VARIABILIDAD COMO ACCIÓN ESTRATÉGICA DE DESARROLLO?

Para crear resultados de alto rendimiento en los recursos e inversiones existentes en cada organización y proceso, con el control integral de riesgos, es necesario crear un plan de transición y empoderamiento con trabajo colaborativo, en donde es necesario: 
a. Mientras los líderes de las partes interesadas y/o las jefaturas de procesos y organizaciones, con ayuda de sus equipos toman decisiones $\mathrm{y}$ acciones emergentes o remiendan problemas para salir de la crisis y apuros que nunca pensaron que aparecería; y que actualmente en muchos de los casos se han combinado con actos de corrupción y otros factores de riesgo propios de cada uno de los procesos. Que son la causa raíz de esta profunda crisis ambiental, humana y económica; más allá de los riesgos o problemas financieros y tributarios que han pasado a tercer plano por consecuencia de los microorganismos patógenos y la cuarta revolución industrial.

En este sentido, es necesario que los líderes de procesos y organizaciones; como principales inversionistas del bienestar, incursionen en soluciones definitivas; y que financieramente reduciendo gastos por la eliminación de innecesarios o procesos improductivos social y económicamente identificados en el ROI Tasa de Retorno sobre la inversión; o por producto de la liberación de un poco de grasa de sus sistemas. Que servirán para reclutar de la sociedad y mantener a los mejores profesionales con aptitudes académica, física, mental, emocional y social afines a las tareas y al combate de estos fenómenos variables de riesgos ambientales, de seguridad y salud.

b. El equipo multidisciplinar reclutado, conformado por investigadores y gestores prácticos de trabajo colaborativo; debe estar liderado por un Director también con todas las aptitudes comprobadas; que administre y democratice con un SISTEMA OPERATIVO INTEGRAL DE ORGANIZACIONES Y PROCESOS DE ALTO RENDIMIENTO, para en etapas de PLANIFICAR, HACER, VERIFICAR, ACTUAR, MEJORAR solucionen secuencialmente la problemática actual desde la raíz. Acción en la cual los líderes, inversionistas y autoridades deben asumir con la mejor predisposición, para remplazar las acciones temporales adoptadas e improvisadas por las acciones definidas producto del cálculo y contribución del equipo multidisciplinar.

c. Es claro y comprensible en los sistemas integrados de gestión, que todo lo que no se mide causa incer- tidumbre; ya que se desconoce si genera ganancia o pérdidas. Con el propósito de controlar desde los orígenes agresivos efectos o riesgos de la variabilidad del entorno; es imprescindible; medir en tiempo real y estadísticamente con la mejor tecnología libre y segura existente de internet, la gestión de la calidad del trabajo y riesgos de todos los procesos sin excepción. En etapas de PLANIFICACIÓN, ACCIÓN, VERIFICACIÓN, CONTROL, MEJORA. Que enfocan a cumplir con herramientas desconcentradas de software libre en internet y un sistema operativo integrado de organizaciones y procesos de alto rendimiento, requisitos: de las partes interesadas; legislación y requisitos de los estándares internacionales, OIT, OMS, OMC, ISO, otros aplicables.

Con plenas capacidades de control y correcciones de todo el capital humano en cuanto a desviaciones de la calidad y el control integral de la variabilidad de riesgos; en cortos plazos de tiempo. A los que todos se exponen por factores humanos. Tal es el caso, la medición estadística de:

- Procesos, cadena de valor, cadena de control, cadenas de suministro y stakeholders.

- Inversionistas, socios, accionistas, promotores.

- Estrategas, administradores, gestores operativos, gestores de inspección y auditoría, staff.

- Equipo técnico, médico, psicológico, brigadistas y demás colaboradores del sistema de seguridad y salud ocupacional.

- Organismos paritarios, delegados, trabajadores en general.

Esta crisis ambiental, humanitaria y económica, es el mejor escenario para poner en acción el conocimiento y capacidad del capital humano, procesos y organizaciones para demostrar a la sociedad, como aprovechan y usan los recursos e inversiones existentes con ALTO RENDIMIENTO y sin desperdicios; en búsqueda de conseguir sobrevivencia, conocimiento y crecimiento sustentable y sostenible ante estas nuevas amenazas ambientales, humanas y económicas. Así como para ayudar a activar o reactivar de forma definitiva las sólidas bases de sistemas estructurados de calidad en las organiza- 
ciones y procesos a prueba de cualquier tipo de riesgos, que amenazan de forma cambiante sus estructuras. Recordando siempre que:

Las partes interesadas, el capital humano, y ambiente; son y serán siempre los más aventajados y beneficiados por el nivel sigma de la calidad en el trabajo colaborativo ejecutado con seguridad, salud, educación y competitividad. Considerando que el bienestar, la Seguridad y salud ocupacional, acorde el índice de gravedad de riesgos. Fortalecen la productividad y competitividad con rentabilidad social y económica a cualquier nivel, escenario y con reacción en cadena.

\section{CONCLUSIONES}

- La falta o ineficiente sistema de control integral de la variabilidad de riesgos; han creado y siguen creando más problemas, la falta de métodos y técnicas desconcentradas y adaptadas a cada proceso para solucionarlos crean aún más problemas y crisis social y económica.

- El control integral de la variabilidad de riesgos, garantiza la calidad de la salud y la seguridad del trabajo; mientras la salud y el trabajo son asegurados, se definen el nivel de productividad. Todo este juego de variables en secuencia, determinan la condición del bienestar y economía del ser humano. En tal sentido al ser el bienestar y la economía el resultado de la calidad de la salud y seguridad del trabajo. A menor riesgo mayor calidad de vida y una segura rentabilidad social y económica sustentable.

- Esta crisis es el mejor escenario para poner en acción el conocimiento y capacidad del capital humano y demostrar a la sociedad, como aprovechan y usan los recursos e inversiones existentes con alto rendimiento y sin desperdicios; en búsqueda de conseguir sobrevivencia, conocimiento y crecimiento sustentable y sostenible ante estas nuevas amenazas ambientales, humanas y económicas. Así como para ayudar a activar o reactivar de forma definitiva las sólidas bases de sistemas estructurados de calidad en las organizaciones y procesos a prueba de cualquier tipo de riesgos, que amenazan de forma cambiante sus estructuras. Recordando siempre que: Las partes interesadas, el capital humano, y ambiente; son y serán siempre los más aventajados y beneficiados por el nivel sigma de la calidad de la seguridad, salud, educación y competitividad.

- El bienestar, la Seguridad y salud ocupacional, acorde el índice de gravedad de riesgos; fortalecen la productividad y competitividad con rentabilidad social y económica a cualquier nivel, escenario y con reacción encadenada.

- Todo lo que no se mide causa incertidumbre; ya que se desconoce si genera ganancia o pérdidas. Con el propósito de controlar desde los orígenes agresivos efectos o riesgos de la variabilidad del entorno; es imprescindible; medir en tiempo real y estadísticamente con la mejor tecnología libre y segura existente de internet, la gestión de la calidad del trabajo y riesgos de todos los procesos sin excepción. En etapas de planificación, acción, verificación, control, mejora. Que enfocan a cumplir con herramientas de software libre en internet y un sistema operativo integrado de organizaciones y procesos de alto rendimiento, requisitos: de las partes interesadas; legislación y requisitos de los estándares internacionales, OIT, OMS, OMC, ISO, otros aplicables. Con plenas capacidades de control y correcciones de todo el capital humano en cuanto a desviaciones de la calidad y el control integral de la variabilidad de riesgos; en cortos plazos de tiempo.

- Se expone por factores de calidad, control de riesgos y humanos a investigación y/o mediciones y/o evaluaciones y/o planes de mejora periódicas de tipo estadístico, todo:

- Procesos, cadena de valor, cadena de control, cadenas de suministro y stakeholders.

- Inversionistas, socios, accionistas, promotores.

- Estrategas, administradores, gestores operativos, gestores de inspección y auditoría, staff.

- Equipo técnico, médico, psicológico, brigadistas y demás colaboradores del sistema de seguridad y salud ocupacional.

- Organismos paritarios, delegados, trabajadores en general. 
- La creciente rentabilidad social y económica se da por las condiciones de bienestar, salud y seguridad ocupacional de la población de interés. Jamás y sin excepción alguna, por naturaleza humana un trabajador o población económicamente activa, enferma o con verdaderas amenazas de riesgos y peligros a su integridad física, mental o social; va a contribuir con alto rendimiento a su sistema de dependencia; por el contrario, éste se torna en otro factor agresivo de riesgo y pérdidas para su ecosistema.

- El único camino recto y sin pérdidas de tiempo ni recursos para superar etapas de crisis, problemas y riesgos; es elevar al máximo rendimiento los recursos existentes. Así como controlar secuencialmente en orígenes los riesgos, problemas y desperdicios de mayor índice de gravedad, de manera sostenible en el tiempo. Con un sistema integrado de gestión de la calidad y anti soborno, que persigan bajo estamentos legales y estándares internacionales; ser altamente rentables social y económicamente para inversionistas y partes interesadas. Esta obligación es única y exclusiva para procesos y organizaciones públicas y privadas de alto rendimiento, Los demás toman sus propias decisiones de sobrevivencia

- Para planificar, hacer, mejorar, controlar, actuar en sistemas de alto rendimiento de recursos e inversiones que nos lleve a superar la crisis ambiental, humanitaria y económica; así como para identificar, medir, evaluar, controlar procesos. Son primeramente necesarios antes de actuar, aplicar la investigación en: identificar las verdaderas necesidades y requisitos de cliente y partes interesadas; que se suman requisitos legales y estándares internacionales en todos los procesos y escenarios; y que los domina el talento humano con plenas aptitudes académica, física, mental, emocional, social; afines a las tareas que ejecutan. Para garantizar rentabilidad social y económica con verdadera calidad lean six sigma y bajo índice de gravedad de riesgos.

- Las partes interesadas, son y serán siempre los más aventajados por su nivel de competitividad. Mientras los demás perderán incluso su tranquilidad, empleos y recursos. Considerando que el bienestar, la Seguridad y salud ocupacional, están directamente consolidados en un único Sistema Operativo de Organizaciones y procesos de alto rendimiento, Software libre de internet KHMCR BI GRUSAM; así como sistémicamente están correlacionados y dependientes de al menos los requisitos de las partes interesadas; marco legal aplicable; estándares internacionales ISO para los sistemas integrados de gestión de la calidad; diseño, ejecución y monitoreo de estrategias propias para la implementación y sostenimiento en el tiempo de una cultura organizacional de alto valor; capital humano con aptitudes; métodos científicos y empíricos generadores de resultados; trabajo colaborativo sin discriminación, entre otros requerimientos propios de cada proceso y realidad.

- Implementando el control integral de la variabilidad de riesgos; en la era de los micro organismos patógenos y cuarta revolución industrial. Seguramente dejaremos de pensar, que un representante legal; profesionales; Delegados de los Organismos Paritarios. Ya no son suficientes para hacer frente a la variabilidad y agresividad de los factores de riesgos en un ámbito integral, que inician a aparecer con el COVID 19, ni siquiera de un grupo de 5 trabajadores, que operativizan sus labores en un mismo centro de trabajo. Peor aún, si se encuentren colaborando dispersos geográficamente. Cuando tenemos plena conciencia como profesionales que los riesgos biológicos y el impacto psicosocial se tornan en incontrolables, si continuamos utilizando técnicas y métodos tradicionalistas y esperando que las charlas, las mascarillas, los guantes los reportes reactivos, proactivos, taza de riesgos, reportes a organismos de control; así como el trabajo monólogo de personas. Salven vidas humanas, en el mundo laboral.

- Los sistemas laborales en el mundo, que acertadamente se han involucrado para desarrollarse en la cuarta revolución industrial; cada vez se fortalecen en su rentabilidad social y económica por la inteligencia artificial, robotización, sistematización de procesos y capital humano con plenas aptitudes; sin quedarse exenta de afectaciones ambientales y económicas por el impacto de la variabilidad. Mientras los trabajos, grupos vulnerables y su capital humano en especial aquellos que tienen adicciones, trastornos vasculares, sistema inmunitario debilitado, asma, car- 
diopatías, cáncer, entre otras alteraciones de la salud; son las próximas víctimas del impacto y agresividad de los factores de riesgos vinculados.

\section{RECOMENDACIONES}

- Esta crisis ambiental, humanitaria y económica, es una fuerte razón, para que todos los profesionales sin excepción y desde cualquier espacio, meditemos, investiguemos y desarrollemos de forma desconcentrada, con alta calidad lean six sigma en búsqueda de los 3,4 errores por cada millón de actividades y trabajo colaborativo. Desde la planificación, acción hasta los resultados. Soluciones y servicios para que todo ser humano en etapa de educación, formación, trabajo, productiva, sustento familiar; así como autoridades, gobiernos inversionistas, colegas, trabajadores; Aunemos esfuerzos y hagamos frente con inteligencia competitiva a tan poderos factores de riesgo como los que comienzan en aparecer en épocas de los microorganismos patógenos y cuarta revolución industrial.

- Para controlar con planificación y dirección estratégica los graves riesgos y efectos a la vida, salud, seguridad y por consecuencia a la economía y productividad que vive la sociedad actualmente. Es necesario que toda la sociedad genere proyectos de vida, basados en su disponibilidad de aptitudes y recursos; sin sueños, sino con objetivos claramente definidos y con todo el recurso alineado a ellos; siempre que se adapten a la realidad ambiental actual. El mundo sin microorganismos agresivos como el CORONAVIRUS, SARS-COV2 ENTRE OTROS seguirán apareciendo en el tiempo y muy difícilmente en la actual y siguientes generaciones, estarán preparadas inicialmente las vacunas o tratamientos médicos para controlar sus efectos. El ecosistema tiene más de Un millón y medio de microorganismos y éstos siguen cambiando y mutando en el tiempo.

- La activación o reactivación de la sociedad, economía de la productividad y competitividad no solo se dan por conseguir abundancia de recursos económicos y materiales; ni tampoco con establecer exoneraciones tributarias, ni solo con aplicar medidas legales, financieras, económicas, comerciales o discursos motivadores. Estas medidas tradicionales y tradicionalistas lo que conllevan es a poner en tela de duda sobre la aptitud de los gestores; así como a malgastar los pocos recursos disponibles en las organizaciones y sociedad; como el caso de agotar recursos económicos, únicamente en el más débil sistema de control de riesgos, como lo representan las compras de EPP, equipos de protección personal. Considerando que la mejor estrategia y acción desde el campo de la seguridad y salud es la erradicación de riesgos en la fuente o en el canal de contagio. Cuando todos sabemos que el único trabajo bien hecho nace de la Planificación, llega a la Acción y genera Resultados favorables, de un equipo multidisciplinar de trabajo con aptitud académica, física, mental, emocional, social. Siempre y cuando con cultura organizacional y trabajo colaborativo se demuestre alto rendimiento en las inversiones y recursos existentes. Caso contrario las inversiones son desperdicios irreparables de recursos.

- Aprendamos ¿Cómo piensa un inversionista en el mundo para mantener, recortar o eliminar recursos?

Simplemente emplea indicadores estadísticos, sobre el rendimiento que es directamente proporcional a la calidad o inversamente proporcional a los riesgos; así como indicadores de Rentabilidad Social y Económica; por ejemplo:

INDICE DE CALIDAD DEL PROCESO. - Relaciona el número de actividades con los fallos o desviaciones, considerando, sigma de la calidad como unidad de media, misma tendencia de calidad que busca el seis sigma que equivale a rendimientos del $99.99997 \%$ en cada millón de procedimientos; e índice de riesgos tendientes al 0\% con control de la variabilidad.

RENTABILIDAD SOCIAL. - Equivale al valor que un proyecto aporta a la sociedad como beneficios, una vez ejecutada y puesta en marcha, el cual puede ser positiva independiente de si su rentabilidad económica RENTABILIDAD ECONÓMICA. - Llamada también ROI mide la capacidad que tienen los activos de una organización para generar beneficios, sin tener en cuenta como han sido financiados. Los beneficios que se tienen en cuenta para conocer la rentabilidad económica son antes de haber descontado los intereses e impuestos correspondientes que la organización tiene que pagar. 


\section{BIBLIOGRAFÍA}

Motivación:

Código Orgánico - Ministerio de Salud Pública. (2016). https://www.salud. gob.ec/wp-content/uploads/2016/11/ RD_248332rivas_248332_355600.pdf

Código de Trabajo ecuador 2020 - Buscar con Google. (n.d.). Retrieved February 11, 2020, from https:// www.google.com/search?ei=0oclYMydPOyb5wKJmqaQCw\&q=Código+de+Trabajo+ecuador+2020\&oq=Código+de+Trabajo+ecuador+2020\&gs_lcp=Cgdnd3Mtd216EAMyAggAMgYIABAHEB4yAggAMgYIABAHEB4yAggAMgUIABCxAzIGCA AQBxAeMgUIABCxAzICCAAyBAgAEB46BwgAELADEEM6BwgAEEcQsAM6BQgAEM0CUPzHBFi-4wRg6-YEaAFwAng AgAHbAogB4geSAQcwLjQuMC4xmAEAoAECo AEBqgEHZ3dzLXdpesgBCMABAQ\&sclient=gwswiz\&ved=0ahUKEwjMzozeyuLuAhXszVkKHQmNCbIQ4dUDCA0\&uact $=5$

Código de la Producción ecuador 2020 - Buscar con Google. (n.d.). Retrieved February 11, 2020, from https://www.google.com/search?ei=jYglYIK-FcTI5gLSOKi4DA\&q=•\%09Código+de+la+Producción+ecuador+2020\&oq=•\%09Código+de+la+Producción+ecuador+2020\&gs_lcp=Cgdnd3Mtd216EAMyBQgAEMOCOgQIIRAKUMHtCVj_9QlgnvwJaABwAngAgAG4AYgB6wOSAQMwLjOYAQCgAQKgAQGqAQdnd3Mtd216wAEB\&sclien$\mathrm{t}=$ gws-wiz\&ved=0ahUKEwjCuPu2y-LuAhVEpFkKHVIoCscQ4dUDCA0\&uact $=5$

Constituyente, A. (2008). Constitución de la República del Ecuador.

Ecuador. (1999). Código de procedimiento civil: legislación conexa (Vol. 2). Corporación de Estudios y Publicaciones.

Códigode Trabajoecuador 2020-Buscar con Google.(n.d.). Retrieved February 11, 2020, from https://www. google.com/search?ei=0oclYMydPOyb5wKJmqaQCw\&q=Código+de+Trabajo+ecuador+2020\&o$\mathrm{q}=$ Código+de+Trabajo+ecuador+2020\&gs_lcp=Cgdnd3Mtd216EAMyAggAMgYIABAHEB4yAggAMgYIABAHEB4yAggAMgUIABCxAzIGCAAQBxAeMgUIABCxAzICCAAyBAgAEB46BwgAELADEEM6BwgAEEcQsAM6BQgAEM0CUPzHBFi-4wRg6-YEaAFwAngAgAHbAogB4geSAQcwLj QuMC4xmAEAoAECoAEBqgEHZ3dzLXdpesgBC MABAQ\&sclient=gws-wiz\&ved=0ahUKEwjMzoz eyuLuAhXszVkKHQmNCbIQ4dUDCAO\&uact=5
Código Orgánico - Ministerio de Salud Pública. (2016). https://www.salud. gob.ec/wp-content/uploads/2016/11/ RD_248332rivas_248332_355600.pdf

Código de Trabajoecuador 2020 - Buscar con Google.(n.d.). Retrieved February 11, 2020, from https://www. google.com/search?ei=0oclYMydPOyb5wKJmqaQCw\&q=Código+de+Trabajo+ecuador+2020\&o$\mathrm{q}=$ Código+de+Trabajo+ecuador+2020\&gs_lcp=Cgdnd3Mtd216EAMyAggAMgYIABAHEB4yAggAMgYIABAHEB4yAggAMgUIABCxAzIGCAAQBxAeMgUIABCxAzICCAAyBAgAEB46BwgAELADEEM6BwgAEEcQsAM6BQgAEM0CUPzHBFi-4wRg6-YEaAFwAngAgAHbAogB4geSAQcwLj QuMC4xmAEAoAECoAEBqgEHZ3dzLXdpesgBC MABAQ\&sclient=gws-wiz\&ved=0ahUKEwjMzoz eyuLuAhXszVkKHQmNCbIQ4dUDCAO\&uact=5

Código Orgánico - Ministerio de Salud Pública. (2016). https://www.salud. gob.ec/wp-content/uploads/2016/11/ RD_248332rivas_248332_355600.pdf

- Código de la Producción ecuador 2020 - Buscar con Google. (n.d.). Retrieved February 11, 2020, from https://www.google.com/search?ei=jYglYIK-FcTI5gLSOKi4DA\&q=•\%09Código+de+la+Producción+ecuador+2020\&oq=•\%09Código+de+la+Producción+ecuador+2020\&gs_lcp=Cgdnd3Mtd216EAMyBQgAEMOCOgQIIRAKUMHtCVj_9QlgnvwJaABwAngAgAG4AYgB6wOSAQMwLjOYAQCgAQKgAQGqAQdnd3Mtd216wAEB\&sclien$\mathrm{t}=$ gws-wiz\&ved=0ahUKEwjCuPu2y-LuAhVEpFkKHVIoCscQ4dUDCA0\&uact $=5$

- Instrumento Andino y Reglamento del Instrumento Andino Decisión 584 - Buscar con Google. (n.d.). Retrieved February 11, 2020, from https://www. google.com/search?q=•+Instrumento+Andino+y+Reglamento+del+Instrumento+Andino+Decisión $+584 \&$ oq $=\bullet \% 09$ Instrumento + Andi no+y+Reglamento+del+Instrumento+Andino+Decisión+584\&aqs=chrome..69i57j69i60.590j0j7\&sourceid=chrome\&ie=UTF-8

Oficial Suplemento. LEY DEL SISTEMA ECUATORIANO DE LA CALIDAD Estado: Vigente PRESIDENCIA DEL CONGRESO NACIONAL Quito, 8 de febrero del 2007 Oficio No. 0258-PCN. Retrieved February 11, 2020, from www.lexis.com.ec

Penal, C. O. I., \& OFICIAL, D. D. R. (2017). Código Orgánico Integral Penal, COIP. 


\section{CONVERGENCE TECH}

\section{Estándares internacionales:}

ISO 10004, Gestión de la calidad - Satisfacción del cliente - Directrices para el seguimiento y la medición

ISO 26000 Sistemas de Gestión de la Responsabilidad Social

ISO 31000 Sistemas de Gestión de Riesgos

ISO 9001 Sistemas de Gestión de la Calidad - Requisitos ISO 14001, Sistemas de gestión ambiental -

Especificación con orientación para su uso

ISO 45001 Sistema de Gestión de Seguridad y Salud en el Trabajo

ISO 27001 ISO/IEC Sistema de Gestión de Seguridad de la Información

ISO 17258 Métodos estadístico - Lean Six Sigma

ISO 10014, Gestión de la calidad - Directrices para la obtención de beneficios financieros y económicos

ISO 10015, Gestión de la calidad - Directrices para la formación, otros 